\title{
ETSI Reconfigurable Radio Systems: Status and Future Directions on Software Defined Radio and Cognitive Radio Standards
}

Markus Mueck, Infineon Technologies

Antti Piipponen and Kari Kalliojärvi, Nokia Research Center

George Dimitrakopoulos, Kostas Tsagkaris, and Panagiotis Demestichas, University of Piraeus

Fernando Casadevall, Jordi Pérez-Romero, and Oriol Sallent, Universitat Politècnica de Catalunya

Gianmarco Baldini, Joint Research Center of the European Commission

Stanislav Filin and Hiroshi Harada, NICT

Merouane Debbah, Supelec

Thomas Haustein, Fraunhofer Heinrich-Hertz-Institute

Jens Gebert, Alcatel-Lucent Deutschland AG

Benoist Deschamps, ANFR

Paul Bender, Bundesnetzagentur

Michael Street, NATO C3 Agency

Sithamparanathan Kandeepan, CREATE-NET

Jaswinder Lota, University of East London

Aawatif Hayar, EURECOM

\begin{abstract}
This article details the current work status of the ETSI Reconfigurable Radio Systems Technical Committee, positions the ETSI work with respect to other standards efforts (IEEE 802, IEEE SCC41) as well as the European Regulatory Framework, and gives an outlook on the future evolution. In particular, software defined radio related study results are presented with a focus on SDR architectures for mobile devices such as mobile phones. For MDs, a novel architecture and inherent interfaces are presented enabling the usage of SDR principles in a mass market context. Cognitive radio principles within ETSI RRS are concentrated on two topics, a cognitive pilot channel proposal and a Functional Architecture for Management and control of reconfigurable radio systems, including dynamic self-organizing planning and management, dynamic spectrum management, joint radio resource management. Finally, study results are indicated that are targeting a SDR/CR security framework.
\end{abstract}

\section{INTRODUCTION}

Cognitive radio (CR) was first identified as a preferred technology for high-end applications in the military and public safety domain when the general CR concept had emerged [1]. Then CR research was also oriented toward the needs of civil wide area (cellular) and short-range communication systems, as demonstrated by early prototypes of institutions such as the Communications Research Center of Canada, and the Shared Spectrum Company, among others. In Europe a series of related collaborative research programs were set up, including IST-E $E^{2} \mathrm{R}$ and II, ICT-E ${ }^{3}$ [2], and more. Early civil CR research was mainly motivated by ensuring an efficient operation of license exempt equipment in the 5 $\mathrm{GHz}$ bands; finally, those studies opened the doors for further investigations in lower frequency bands. The involved industrial, regulatory, and academic partners were attracted to CR by the prospect of a hugely increased level of spectral efficiency and improved overall system capacity exploitation, among others, thanks to: 
- The dual exploitation of spectrum by applying opportunistic spectrum usage

- A mobile device (MD) being (partially/fully) aware of its (heterogeneous) context (radio context, application context, etc.) and dynamically adapting its parameters such that its operational objectives are reached in an optimum way; for example, a MD is aware of surrounding radio access technologies (RATs) and selects those that guarantee to fulfill its quality of service (QoS) requirements at the lowest cost (in terms of subscription cost, power consumption, etc.).

Software defined radio (SDR), on the other hand, is considered to be an enabling technology introducing the required level of flexibility in order to enable a device to adapt to its context.

Following CR related rulemaking by the Federal Communications Commission (FCC) [3], several CR standards are currently under development, mainly within the IEEE and European Telecommunications Standards Institute (ETSI) framework. One of the first IEEE Working Groups (WGs) to consider CR technology was IEEE 802.22, created in 2004 and developing a standard for wireless regional area networks (WRANs) using white spaces in the TV frequency spectrum. Another relevant standard is ECMA-392, published in 2009, which specifies a medium access control (MAC) sublayer and a physical (PHY) layer for personal/portable cognitive wireless networks operating in TV bands. Most recently, a new CR boom set in, and several novel groups were created at the end of 2009. IEEE 802.11af defines modifications to both the 802.11 PHY and 802.11 MAC layers to meet the legal requirements for channel access and coexistence in the TV white space. It is expected that this work will build on IEEE 802.11y results, where an inherent key issue was addressed and resolved in a different context: the Dependent Station Enablement (DSE) mechanism defines how an operator extends and retracts permission to devices to use licensed radio spectrum. IEEE 802.19 , on the other hand, works towards enabling the family of IEEE 802 wireless standards to most effectively use TV white space by providing standard coexistence methods. Furthermore, IEEE SCC41 is developing standards related to dynamic spectrum access networks with a focus on improved spectrum usage. In this framework the IEEE standard 1900.4-2009 defines a management system supporting network-terminal distributed optimization of radio resource usage and improvement in QoS in heterogeneous wireless networks. Further standards are currently in preparation within IEEE SCC41, including P1900.4a for enabling mobile wireless access service in white space frequency bands without any limitation on used radio interface.

The ETSI Reconfigurable Radio Systems (RRS) Technical Committee (TC), on the other hand, is performing work that is complementary to the IEEE SCC41 and IEEE 802 activities, with a focus on the following: SDR standards beyond the IEEE scope, CR/SDR standards addressing the specific needs of the European Regulatory Framework, and CR/SDR TV white space standards adapted to the digital TV signal characteristics in Europe. Further details are given in the

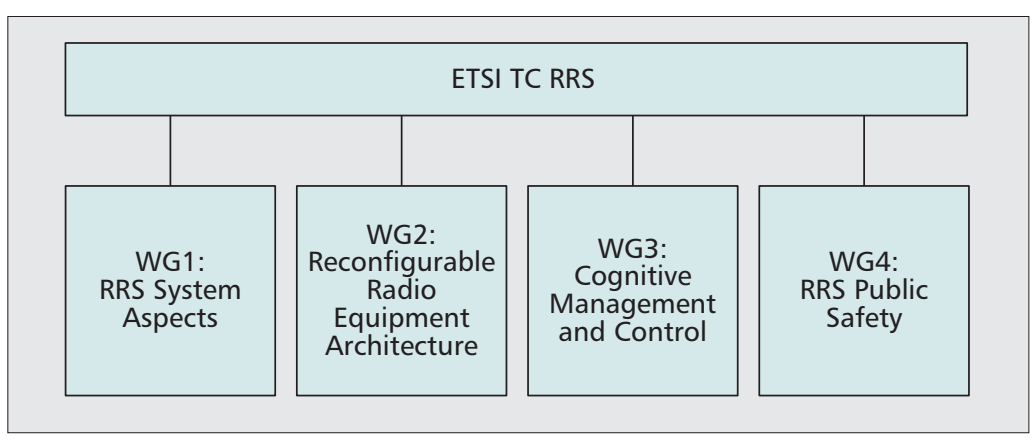

Figure 1. ETSI RRS structure.

sequel to this article. The next section gives a general overview on ETSI RRS and comments on the European Regulatory Framework. We then give a general overview of the ETSI RRS concept and vision. We then detail CR aspects focusing in particular on a cognitive pilot channel (CPC) and functional architecture (FA) for management and control of reconfigurable radio systems. We then present the SDR related study results, currently focusing on an MD SDR architecture and related interfaces. We then highlight security issues addressed in the ETSI RRS framework, while the final section gives an outlook on future topics based on current progress in academic research and a conclusion.

\section{ETSI RRS: OVERVIEW AND ROLE IN THE EUROPEAN REGULATORY FRAMEWORK}

At the inaugural meeting, the ETSI RRS TC created the following four WGs, in which the technical discussions are organized and reports are produced (Fig. 1).

WG1 focuses on system aspects and develops proposals from a system aspects point of view for a common framework in RRS TC with the aim to guarantee coherence among the different RRS TC WGs and to avoid overlapping and gaps between related activities.

$W G 2$ focuses on SDR technology with a particular interest in radio equipment architecture and proposes common reference architectures for SDR/CR radio equipment (mobile handset devices, radio base stations, etc.), related interfaces, and so on.

WG3 focuses on cognitive management and control; the group collects and defines the system functionalities for reconfigurable radio rystems related to spectrum management and joint radio resource management across heterogeneous access technologies. Furthermore, the group has developed a functional architecture for the management and control of reconfigurable radio systems as well as a report on the CPC as an enabler to support the management of the RRS.

WG4 focuses on public safety and collects and defines the related RRS requirements from relevant stakeholders in the public safety and defense domain. The group defines the system aspects for the applications of RRS in public safety and defense. 
Broadcasting,

wireless

microphones, and

assignment to radio

stations are

managed in Europe

at the national level.

Any sharing scheme

based on a database

will require some

level of integration

of the national data.
Building on this structure, ETSI RRS will complement ongoing efforts in other bodies, such as IEEE standardization bodies, by proposing technological solutions beyond the existing scope (related to SDR interfaces, CR-specific management, and control architectures and interfaces, knowledge management via a CPC, and security solutions); furthermore, ETSI RRS fulfills a key role in the framework of European regulation, with a focus, among other aspects, on the following:

- The R\&TTE Directive regime in force in Europe is based on declaration of conformity and includes neither type approval nor registration of the equipment or equipment identifier (in the United States, type approval is still necessary). This self-declaration is preferably a reference to a Harmonized standard to be developed by ETSI RRS.

- Protection of TV bands: In Europe, digital video broadcast - television (DVB-T) does not show a residual carrier as is the case in the United States (the possibility for detection of the U.S. Advanced Television Systems Committee (ATSC) signal below noise (i.e., at $-114 \mathrm{dBm}$ ) is made possible thanks to the residual carrier that is present in the ATSC signal). A corresponding adaptation of sensing-based standards needs to be defined for Europe.

- Broadcasting, wireless microphones, and assignment to radio stations are managed in Europe at the national level. Any sharing scheme based on a database will require some level of integration of the national data.

In order to address the above and other European regulatory aspects, the Electronic Communications Committee (ECC) within the European Conference of Postal and Telecommunications Administrations (CEPT) has set up the SE43 group working on "Technical and Operational Requirements for the Operation of Cognitive Radio Systems in the 'White Spaces' of the Frequency Band 470-790 MHz." ETSI RRS is the competence center within ETSI to implement those regulatory requirements.

\section{ETSI RRS SYSTEM ASPECTS OVERVIEW AND CR VISION}

WG1 has conducted feasibility studies on cognitive radio systems (CRSs) concept and potential regulatory aspects of CRS and SDR. The technical concept of CRS developed by WG1 includes the following key elements:

- Objectives

- Spectrum use scenarios

- Technical requirements

- Spectrum management layers

- Architectural approaches

- Enabling technologies

Several objectives have been identified for CRSs in order to ensure more efficient and flexible use of spectrum. For this purpose, a CRS performs three key activities: obtain knowledge of the radio operational environment and location, decide on the gathered information and act based on this decision, and learn from the results obtained.

Enhancing user experience is one of the main objectives of the CRS. Application examples are cross-operator access, user networks, flexible access to the future internet, and connecting to smart spaces. The CRS is expected to be beneficial for optimization of the mobile operator network. This could include load balancing, spectrum refarming, and radio resource usage optimization.

CRS deployment examples are categorized according to the following four spectrum use scenarios: dedicated spectrum, shared spectrum, secondary usage in dedicated spectrum, and spectrum dedicated for CRSs. The first category includes such scenarios as autonomous reconfiguration of software defined multiradio, and reconfiguration of terminals and base stations in a composite wireless network. Shared spectrum scenarios consider deployment of CRS in license exempt bands. Secondary usage in dedicated spectrum considers a scenario where a CRS shares the spectrum within the current licensed allocations on a secondary basis.

The overall CRS concept developed by ETSI RRS is depicted in Fig. 2. The figure covers both centralized and decentralized solutions for CRs, where the centralized, operator-driven solution is targeted for wide area utilization, and the decentralized solution is targeted for local area ad hoc/mesh networking.

The centralized CRS concept is represented by the composite wireless network (CWN) including cognitive network management system (C-NMS). C-NMS contains such key components as the operator spectrum manager (OSM) and joint radio resource management (JRRM). The decentralized CRS concept is represented by the cognitive mesh network (CMN) controlled by the cognitive control network (CCN).

WG1 has also identified key enabling technologies for the CRS. They include software defined radio and multiradio, reconfigurable base stations management, spectrum sensing, cognitive pilot channel, cognitive control radio and networking, geolocation, primary protection database, and distributed decision making.

\section{ETSI RRS Cognitive Radio Solutions}

In order to address the stringent requirements stemming from the introduction of CR technology, WG3 of ETSI RRS has focused on the management of reconfigurable radio systems (encompassing both SDRs and CRs). Specifically, the group has conducted studies on a functional architecture (FA) for the management of RRS. This comprises the description of the requirements, of the derived functional building blocks, and the key interfaces among them. In addition, the group has refined and further analyzed the CPC concept, which was originally proposed to facilitate the collaboration between network and terminals in heterogeneous radio environments $[4,5]$, and can be seen as an enabler to support the management of RRS.

The group has investigated the feasibility of standardizing the above concepts and eventually produced two technical reports for the FA [6] and the CPC [7]. 


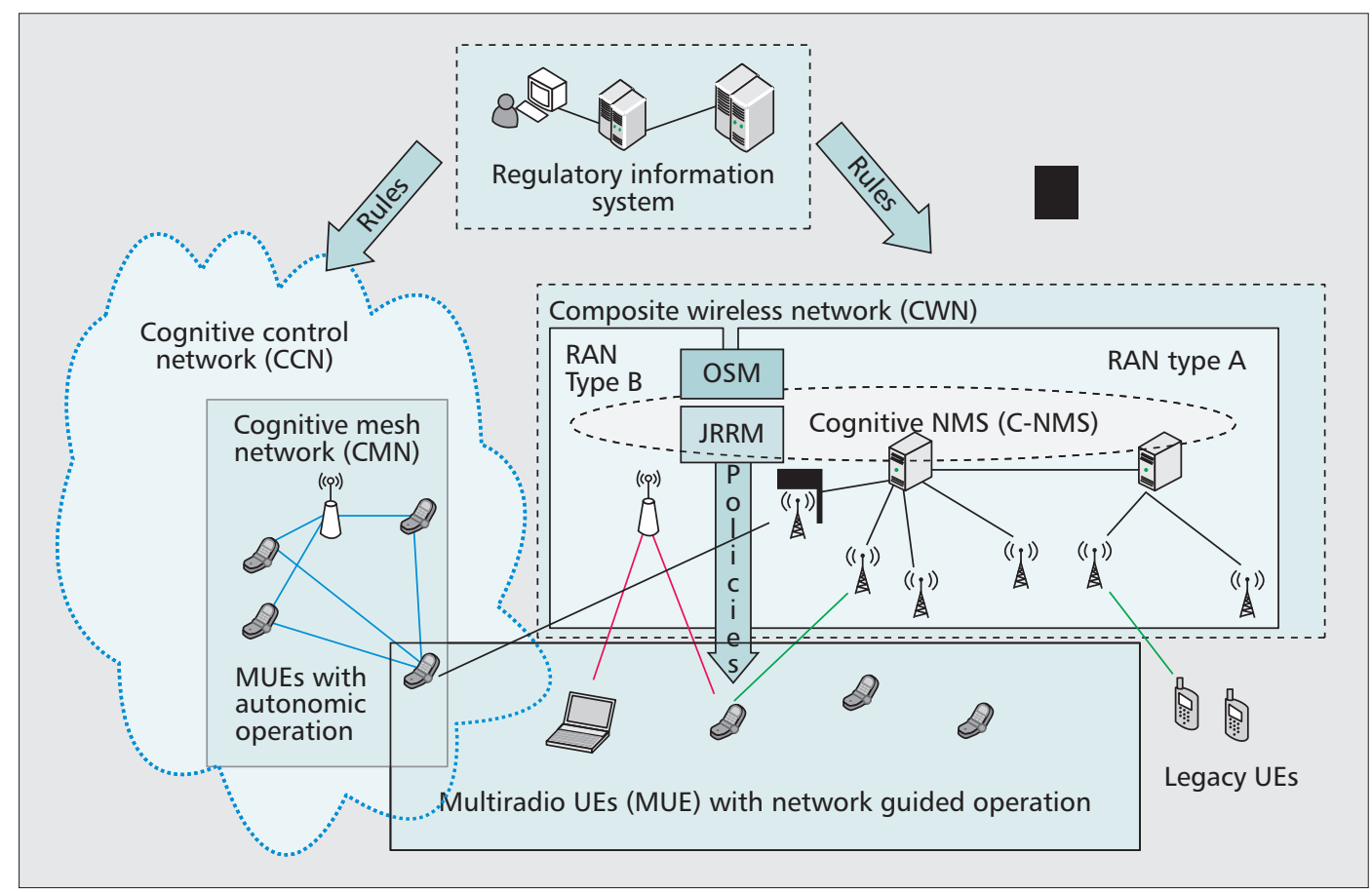

The CPC is defined
as a channel that
conveys the ele-
ments of necessary
information facilitat-
ing the operations of
CRS. The CPC pro-
vides information on
which radio accesses
can be expected in a
certain geographical
area. This informa-
tion includes opera-
tor information, RAT
type as well as used
frequencies.

Figure 2. Centralized and decentralized CR system concepts.

\section{Cognitive Pilot Channel}

The CPC is defined as a channel that conveys the elements of necessary information facilitating the operations of the CRS [4]. The CPC provides information on which radio accesses can be expected in a certain geographical area. This information includes operator information, RAT type as well as used frequencies. Exemplary scenarios where the $\mathrm{CPC}$ is seen as useful are:

- The CPC is used to support a terminal during the startup phase in an environment where the terminal does not yet know the available RATs and corresponding used frequencies.

- In the context of a secondary system, the $\mathrm{CPC}$ is used to exchange sensing information between terminals and base stations in order to perform collaborative/cooperative sensing, facilitating the searching of white spaces to start communication.

- The CPC is used for an efficient level of collaboration between a network and the terminals by supporting radio resource management (RRM) optimization procedures.

While the basic principle of the CPC is shown in Fig. 3, different CPC deployment approaches are possible.

In the out-band CPC solution, where the CPC is conceived as a radio channel outside the component $\mathrm{RAT}$, the $\mathrm{CPC}$ uses either a new radio interface, or alternatively, an adaptation of legacy technology with appropriate characteristics.

In the in-band CPC solution, the CPC is conceived as a logical channel within the technologies of the heterogeneous radio environment.

Furthermore, extensions of the above centralized approaches toward more decentralized ones are currently under discussion, including a distributed cognitive pilot channel (DCPC) [8] and the cognitive control radio (CCR) [9].

\section{FUNCTIONAL ARCHITECTURE FOR MANAGEMENT AND CONTROL OF RECONFIGURABLE RADIO SYSTEMS}

In order to define a functional architecture that is able to provide optimized management of radio and spectrum resources, WG3 of ETSI RRS has collected and reported the following set of requirements:

- Personalization, to support various classes of users

- Support of pervasive computing, enabled by the existence of sensors, actuators, and wireless local area networks in all application areas

- Context awareness, for efficiently handling multiple, dynamically changing, and unexpected situations

- Always best connectivity, for optimally serving equipment and users in terms of QoS and cost

- Ubiquitous application provision for the applications above

- Seamless mobility, for rendering the users agnostic of the heterogeneity of the underlying infrastructure

- Collaboration with alternate RATs for contributing to the achievement of always-best connectivity

- Scalability, for responding to frequent context changes

Accordingly, in order to address these requirements, a proper functional decomposition has been proposed in [6]. The derived functional blocks, together with the interfaces among them and their distribution between network and terminals, is depicted in Fig. 4. The dynamic spectrum management (DSM) block is responsible for the medium and long term, both technical and economical, management of spectrum, and as such it incorporates functionalities like provi- 


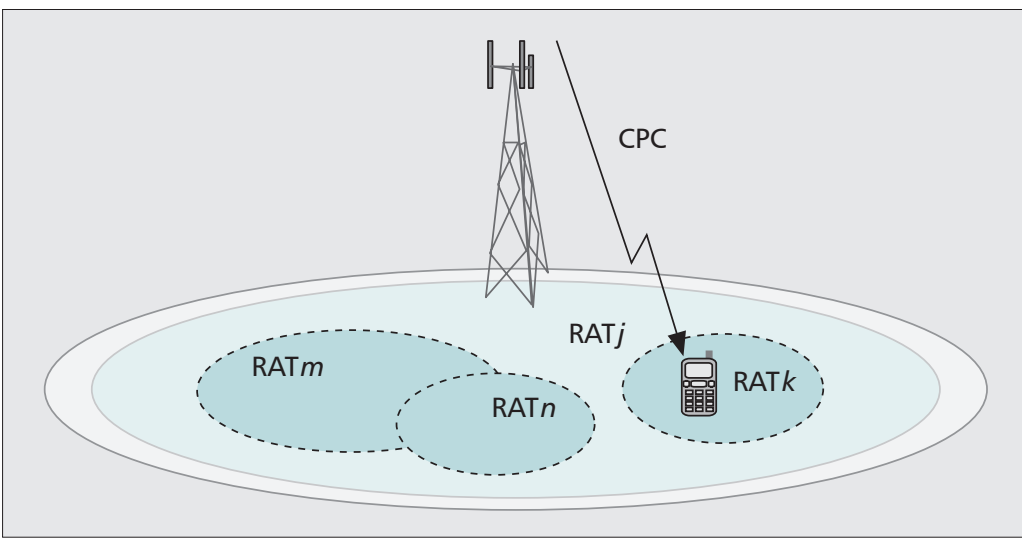

Figure 3. The CPC in a heterogeneous RAT environment.

sioning of information for spectrum assignments, spectrum occupancy evaluation and decision making on spectrum sharing/trading.

Dynamic self-organizing planning and management (DSONPM) caters for the mediumand long-term management at the level of a reconfigurable network segment (e.g., incorporating several BSs). It provides decision making functionality for QoS assignments, traffic distribution, network performance optimization, RATs activation, configuration of radio parameters, and so on. The fundamental objective of the joint radio resources management (JRRM) block is the joint management of radio resources possibly belonging to heterogeneous RATs, and its functionalities mainly include radio access selection, neighborhood Information provision, and QoS/bandwidth allocation/admission control. Finally, configuration control module (CCM) is responsible for the enforcement of the reconfiguration decisions typically made by the DSONPM and JRRM.

\section{ETSI RRS SOFTWARE DEFINED RADIO SOLUTIONS}

ETSI RRS considers SDR related standardization for both base stations (BSs) and MDs. The BS related work is currently in an early stage and available results are presented in [10]. The current focus in ETSI RRS WG2 relies mainly on MD SDR related interface standardization between distinct stakeholder domains, such as SDR chipset vendors and MD manufacturers. In this framework a reference architecture has been derived, which outlines the relevant interfaces and concerned building blocks - this architecture, however, is not meant to be normative.

\section{AN SDR ARCHITECTURE APPROACH FOR MOBILE DEVICES AS A BASIS FOR FUTURE SDR STANDARDS}

ETSI RRS has identified a set of requirements related to an SDR MD architecture [11], including general architectural requirements, capability requirements, operational requirements, interface requirements, and other requirements. The capability requirements are highlighted below.

- Multiradio configuration capability: SDR equipment in an MD is expected to install, load, and activate a radio application while already running a set of radio systems.

- Multiradio operation capability: SDR equipment in an MD is expected to execute a number of radio systems simultaneously by taking into account temporal coexistence rules designed for their common operation.

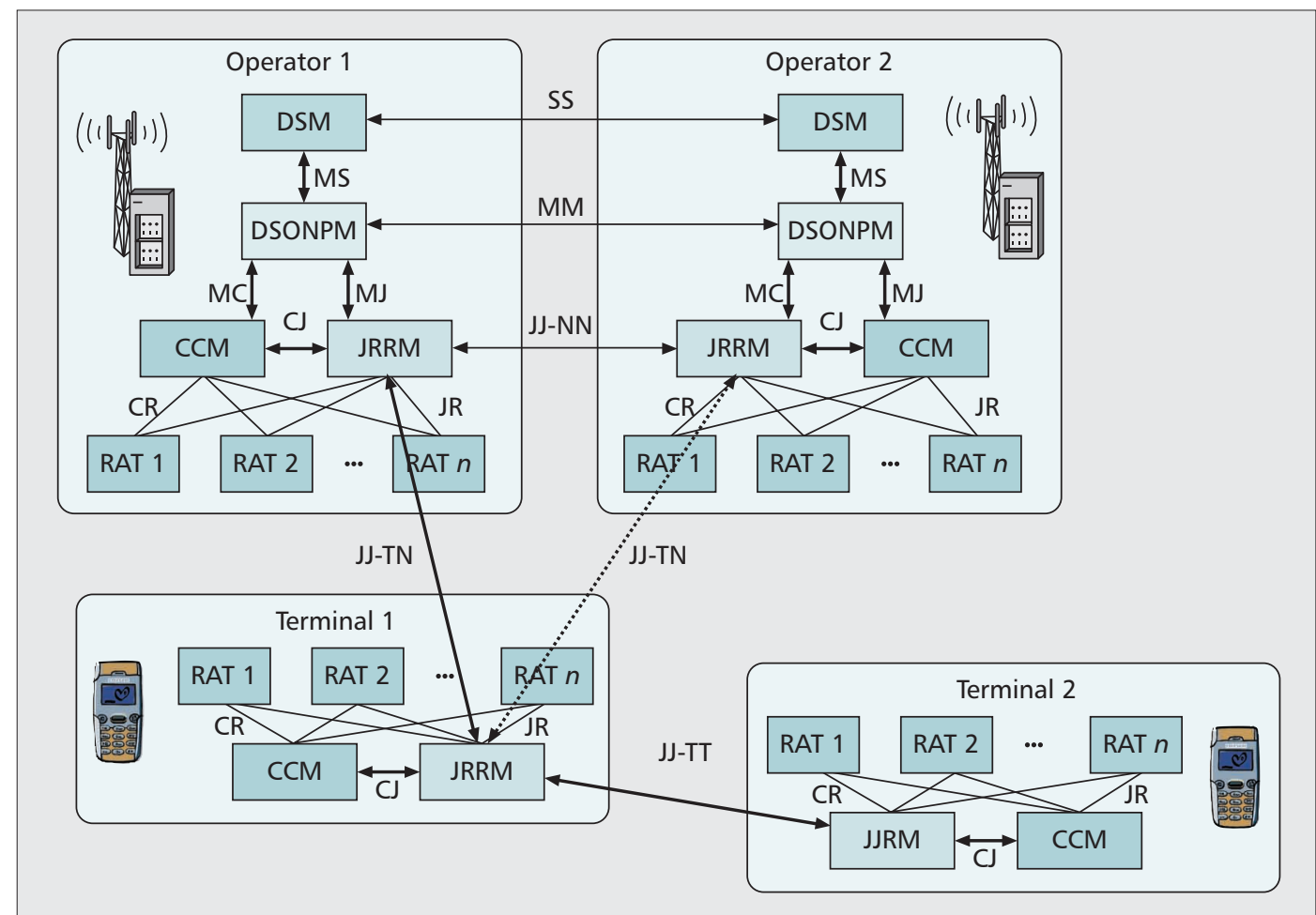

Figure 4. High-level view of the proposed ETSI RRS functional architecture. 

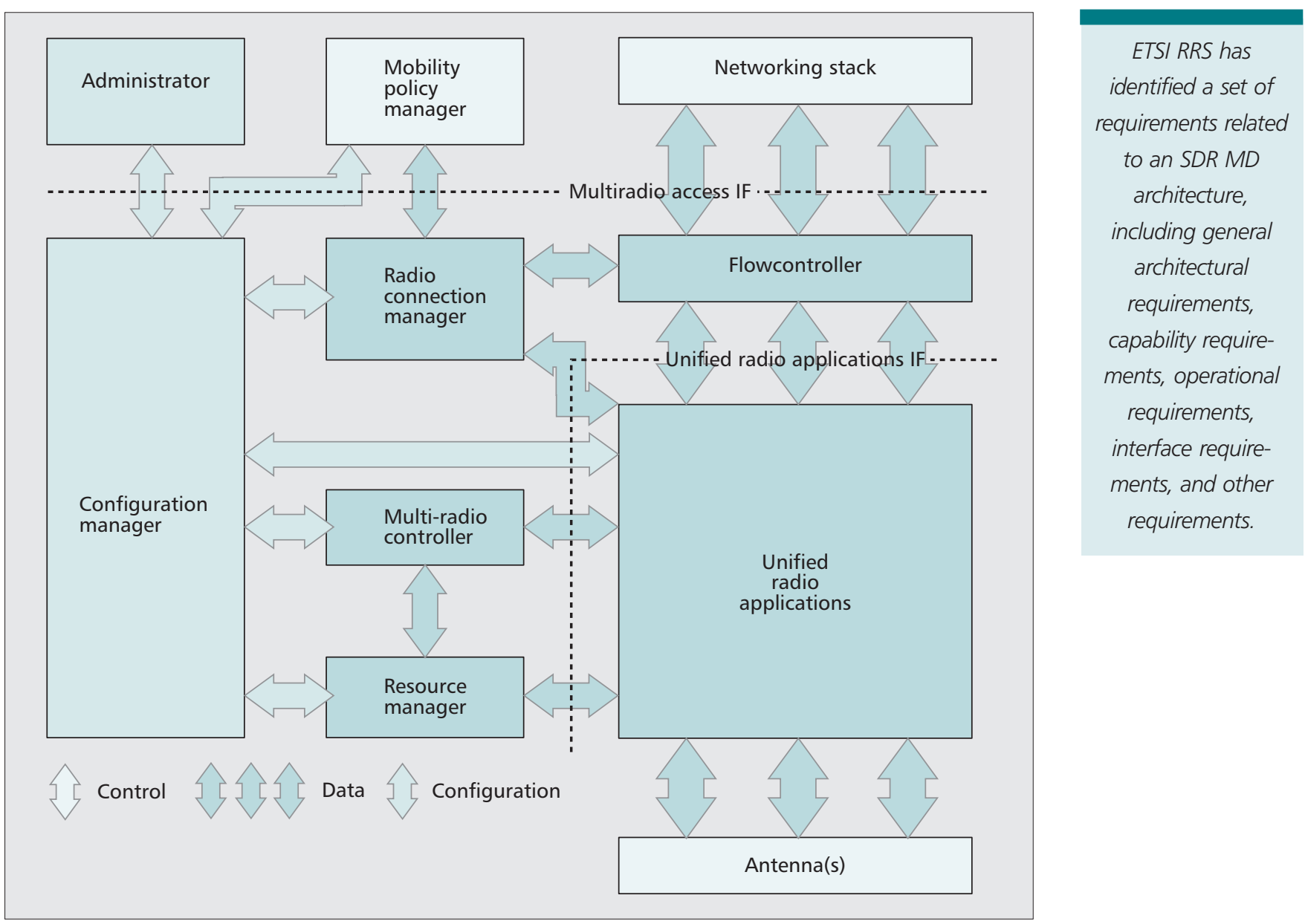

Figure 5. Functional architecture of SDR equipment.

- Multiradio resource sharing capability: SDR equipment in an MD is expected to execute a number of radio systems simultaneously by sharing computation, memory, communications, and RF circuitry resources available on the radio computer platform by using appropriate resource allocation, binding, and scheduling mechanisms.

The outcome of the study consists, among others, of the presentation of a functional architecture for SDR equipment as detailed in Fig. 5.

The components of this framework have different responsibilities as follows:

- Configuration manager: (De)installation and (un)loading of radio applications into a radio computer, as well as management of and access to the radio parameters of those radio applications

- Radio connection manager: (De)activation of radio applications according to user requests and overall management of user data flows

- Flow controller: Sending and receiving of user data packets and controlling the flow

- Multiradio controller: Scheduling the requests on spectrum resources issued by concurrently executing radio applications in order to detect in advance the interoperability problems between them

- Resource manager: Management of radio computer resources in order to share them among simultaneously active radio applications, while guaranteeing their real-time requirements

\section{SDR STANDARDIZATION RELATED TO INTERFACES}

The ETSI RRS WG2 SDR handset reference architecture report [11] identifies four candidate interfaces for standardization:

- Multiradio interface as the uniform interface for network protocol stacks and other user domain entities to access services of the radio computer

- Unified radio application interface at the boundary between the common radio computer platform and the specific radio applications

- Radio programming interface including software development-time concepts and runtime interfaces between radio software entities and the radio computer platform

- Interface to the reconfigurable $R F$ transceiver to support multiple radio applications, even concurrently

Among these interfaces, the multiradio interface has most potential for standardization, and is currently under further studies in ETSI RRS.

The deployment of the multiradio interface is expected to proceed in phases with platform capability advances, starting from legacy radio 


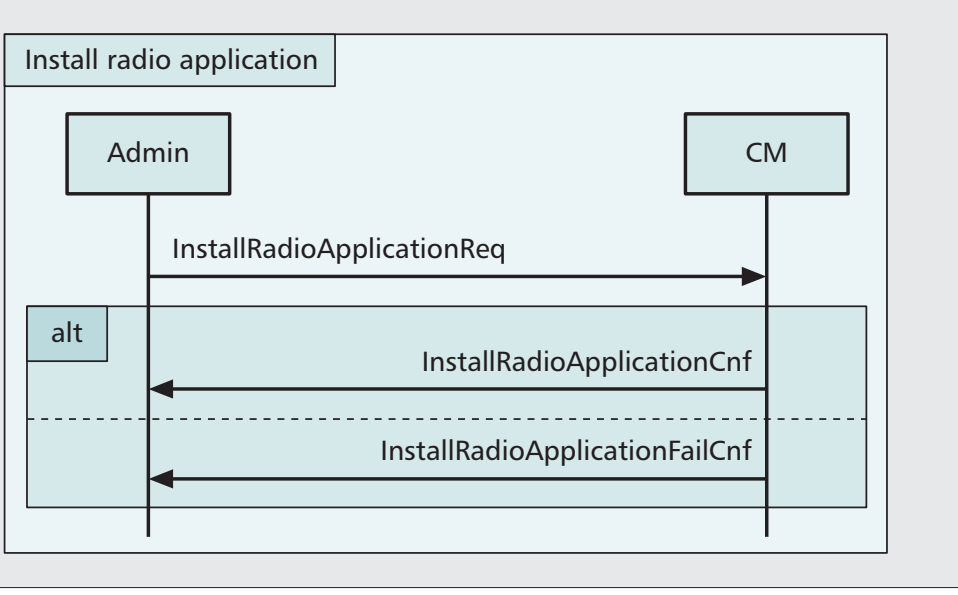

Figure 6. Definition of a service in MURI - the administrator user requests installation of a new radio application from the configuration manager in the SDR platform.
The information model and service definition for standardization pre-study is ongoing in ETSI RRS. The next step after that is to gather feedback from relevant stakeholders such as chipset and mobile device manufacturers to determine if there is sufficient interest to begin actual standardization of the multiradio interface.

\section{SECURITY}

As a general rule, RRS must validate communication security requirements like data confidentiality and privacy, availability, registration, authentication, and authorization already defined for conventional wireless communication systems. RRS may also be vulnerable to new types of security attacks, beyond the ones already identified for conventional networks.

The SDR reconfiguration capability, as described in the previous section, where a radio application can be loaded and activated at runtime could be exploited by a security attacker to download and activate malicious software modules.

To protect the SDR against these types of attacks, we suggest adopt software assurance processes and functions including a software certification processes, a secure download mechanism, which guarantees the authenticity of the downloaded software, and a secure execution environment in the SDR terminal to guarantee that only trusted software can be activated and executed.

With reference to the functional architecture of SDR equipment described in the previous section, these functions could be implemented by a software download authentication (SDA) component, which can be part of the administrator and configuration manager blocks. SDA may use security functions on the radio, which must be implemented with a suitable level of trust. We may have three levels of interfaces to radio resources: public group managed, and national (government controlled).

ETSI TC RRS is also investigating security vulnerabilities and threats of cognitive radio. Conventional communication systems can only change their transmission parameters and use the RF spectrum bands in the limits implemented in their hardware and firmware architectures. A cognitive radio could instead communicate in a wide range of spectrum bands and change its transmission parameters at runtime, on the basis of sensed radio spectrum environment or information received from other cognitive radio nodes. Because of these capabilities, cognitive radio nodes could increase the risk of harmful wireless interference if the cognitive radio mechanism is disrupted or abused.

As described earlier, cognitive radio networks can be based on the concept of CPC, which can become a vulnerability point to denial of service attacks like traffic overload or jamming. Overflow of the CPC can be controlled through algorithms implemented in cognitive radio nodes to analyze repetition of cognitive control messages, while jamming of CPC can be mitigated by defining a number of CPC in various frequency bands, which could be changed if harmful interference is detected.

The exchange of cognitive control messages could be protected by a distributed authentication protocol and a secure encapsulation proto- 
col to guarantree the sources of cognitive messages and their content respectively. In this area security solutions designed for the distribution of routing information in mobile ad-hoc networks could be adapted to the exchange of cognitive messages in cognitive radio networks.

\section{OUTLOOK ON FUTURE ETSI RRS TOPICS AND CONCLUSION}

Different research lines have been initiated from the academic perspective addressing ETSI RRS aspects. Part of this research is also linked to specific projects such as ICT-E ${ }^{3}[2]$. In the sequel current research trends are highlighted, which have the potential to become relevant for ETSI RRS and CR/SDR standardization in general.

As far as the algorithmic level is concerned, specific algorithms for autonomous RAT selection using advanced tools of game theory, stochastic analysis, and decentralized resource allocation are being studied. The cognitive concepts are exploited to carry out dynamic spectrum assignment based on reinforcement learning mechanisms in order to achieve efficient spectrum usage in the context of next-generation mobile flexible networks based on orthogonal frequency-division multiplexing (OFDM).

Spectrum sensing has also become a full dedicated research topic in order to continuously monitor and learn the radio environment. The cognitive radio node senses the spectrum to detect any incumbent spectrum users (ISUs) to opportunistically utilize it in the spatial, temporal and frequency domains. The key term to be considered here is reliably detecting the ISU as per the regulatory requirements.

Current directions for spectrum sensing involve localization techniques to generate a radio environment map (REM) and keep track of the ISU in a spatio-temporal manner.

As far as the coexistence of primary and secondary networks, several spectrum measurement campaigns have already been performed in diverse locations in Europe and have shown the potential of exploiting free unused bands. This has permitted the capacity of cognitive networks to be determined through the analysis of the spectrum occupancy degree of the allocated spectrum bands in real wireless communication systems. Some specific time and frequency domain models of the spectrum usage have also been derived.

Researchers from academia are in fact discussing beyond the cellular scenario, considering more flexible radio communication like peer-topeer or private femto or hotspot concepts. All these approaches have in common that neither the precise spectrum usage nor the air interface parameters are known in advance, such as using a Long Term Evolution (LTE)-like air-interface in industrial, scientific, and medical (ISM) spectrum, provided that general coexistence rules on interference power are fulfilled. Obvious advantages of such a approaches are multi-user capabilities, embedded broadcast, and concepts of interference handling, system bandwidth scalability, and existing frequency-division multiplexing (FDD) and time-division multiplexing (TDD) options.

To give a conclusion, it can be stated that this article has illustrated the SDR and CR framework elaborated by ETSI RRS for a heterogeneous wide-area (cellular) and short-range system scenario. While these studies are reaching a final stage within ETSI RRS, the next step consists in elaborating corresponding normative standards: From December 2009 on, the group is mandated to produce normative standards in the field of CR and SDR (instead of study reports). The existing study results are expected to provide a broad basis for that effort.

\section{ACKNOWLEDGMENT}

While the contents of this article represent the views of the authors, an acknowledgment is given to all ETSI RRS members and contributors who helped to set up this effort.

\section{REFERENCES}

[1] J. Mitola III, Cognitive Radio, Ph.D. thesis, KTH, Stockholm, Sweden, 2000.

[2] ICT-2007-216248, "End-to-End Efficiency $\left(E^{3}\right)^{\prime}$; http://www.ict-e3.eu/

[3] FCC, "Cognitive Radio Technologies Proceeding (CRTP)," ET Docket No. 03-108; http://www.fcc.gov/oet/cognitiveradio/

[4] IST-E2R II White Paper, "The E2RII Flexible Spectrum Management (FSM) Framework and Cognitive Pilot Channel (CPC) Concept - Technical and Business Analysis and Recommendations."

[5] J. Pérez-Romero et al., "A Novel On-Demand Cognitive Pilot Channel enabling Dynamic Spectrum Allocation," IEEE DYSPAN '07, Apr. 17-20, 2007.

[6] ETSI TR 102 682, "Reconfigurable Radio Systems (RRS); Functional Architecture for Management and Control of Reconfigurable Radio Systems," 2009.

[7] ETSI TR 102 683, "Reconfigurable Radio Systems (RRS); Cognitive Pilot Channel (CPC)," 2009.

[8] Sendora, "Report on WSN Aided Cognitive Radio Platform," project deliverable, Dec. 2009.

[9] ETSI TR 102 802, "Reconfigurable Radio Systems (RRS); Cognitive Radio System Concept," 2009.

[10] ETSI TR 102 681, " Reconfigurable Radio Systems (RRS); Radio Base Station (RBS) Software Defined Radio (SDR) Status, Implementations, and Costs Aspects, including Future Possibilities," 2009.

[11] ETSI TR 102 680, "Reconfigurable Radio Systems (RRS); SDR Reference Architecture for Mobile Device," 2009.

\section{BIOGRAPHIES}

MARKUS MUECK [M] (MarkusDominik.Mueck@infineon.com) received his diploma degrees from the University of Stuttgart, Germany, and the Ecole Nationale Supérieure des Télécommunications (ENST), Paris, France, in 1999, and a doctorate degree from ENST Paris in 2006, and became an adjunct associate professor of Macquarie University, Sydney, Australia, in 2009. He was a senior staff member and technical manager at Motorola Labs, Paris, France, from 1999 to 2008. Since 2008 he is standardization manager at Infineon Technologies, Munich, Germany, Chairman of ETSI RRS, and head of delegation for IEEE $802.16 \mathrm{~m}$.

ANTTI PIIPPONEN received his M.Sc. degree from Tampere University of Technology, Finland, in 2004. He currently holds a senior researcher position at Nokia Research Center, and has been working mainly with radio frequency system design, baseband signal processing, and software defined radio.

GEORGE DiMITRAKopoulos received a Diploma in electrical and computer engineering and a Ph.D. degree from the National Technical University of Athens, Greece and the University of Piraeus, Greece, respectively. He is currently working as an adjunct lecturer and senior research engineer at the Department of Digital Systems of the University of Piraeus. He has been actively involved in numerous national and international research projects $\left(E^{3}, E^{2} R\right.$ IMPULSE, ARIADNE, WIN-HPN, DIOSKOUROI). His research interests and activities include the design and management of B3G systems, cognitive systems, optimization algorithms, and queueing theory.
Researchers from

academia are

discussing beyond

the cellular scenario,

considering more

flexible radio com-

munication like peer-

to-peer or private

femto or hotspot

concepts. All these

approaches have in

common that neither

the precise spectrum

usage nor the

air-interface

parameters are

known in advance. 
KOSTAS TSAgKaris [M] received his Diploma and Ph.D. in electrical and computer engineering from National Technical University of Athens. Currently, he is adjunct lecturer and senior research engineer at the Department of Digital Systems of the University of Piraeus. He has published more than 70 papers in the areas of design, management, and optimization of wireless/wired communication networks. He serves as Technical Editor in the IEEE SCC 41/1900.4 Working Group.

Panagiotis Demestichas [M] holds a Diploma and Ph.D. degree in electrical and computer engineering from the National Technical University of Athens. Currently, he is associate professor in the department of Digital Systems of the University of Piraeus. He is the technical manager of the FP7 $E^{3}$ project and the chairman of WG6 ("Cognitive Wireless Networks and Systems") of the Wireless World Research Forum (WWRF). His research interests include the design, management, and performance evaluation of mobile and broadband networks, algorithms, and queueing theory.

JeNS GEBERT received his Diploma in electrical engineering in 1993 from the University of Stuttgart. In the same year, he joined Alcatel as a software engineer for Mobile Systems. He currently works for Alcatel-Lucent in Bell Labs Germany in the Wireless Access Domain on Cognitive Radio Systems. $\mathrm{He}$ is strongly involved in the architecture work within different EU FP7 projects as well as in ETSI TC RRS standardization activities.

Fernando CaSADEvall is a full professor at the Signal Theory and Communications Department of the Universitat Politècnica de Catalunya (UPC), Barcelona, Spain. His research interest is focused in the field of mobile communication systems, especially in cognitive radio issues. He has been involved in fifteen research projects founded by the European Commission and served as a consultant for a number of private companies. He has published around one hundred and fifty of technical papers in both international conferences and magazines.

ORIOL SALLENT is an associate professor at UPC. His research interests are in the field of mobile communication systems, especially radio resource and spectrum management for heterogeneous networks. He has published more than 150 papers in international journals and conferences. He has participated in several research projects of the 5th, 6th, and 7th Framework Programmes of the European Commission, and served as a consultant for a number of private companies.

JORDI PÉREZ-ROMERO is currently associate professor at the Department of Signal Theory and Communications of UPC. His research interests are in the field of mobile communication systems, radio resource and QoS management, and cognitive radio networks. He has been involved in different European projects as well as projects for private companies. He has published papers in international journals and conferences and has co-authored one book on mobile communications

GIANMARCO BALDINI completed his degree in 1993 in electronic engineering from the University of Rome "La Sapienza" with specialization in wireless communications. He has worked as senior technical architect and system engineering manager in Ericsson, Lucent Technologies, Hughes Network Systems, and Selex Communications before joining the Joint Research Centre of the European Commission in 2007 as scientific officer. His current research activities focus on communication services for public safety, security aspects of GNSS services, and modeling of critical infrastructures.

STANISLAV FILIN [SM] is an expert researcher with NICT Japan. He is currently serving as NICT representative in ITUR WP 5A and WP 1B, and ETSI TC RRS. He is a voting member of IEEE SCC 41 and IEEE 1900.4 WG, where he has been serving as Technical Editor and Chair of several subgroups. He was a voting member of IEEE 1900.6 WG. He participated on the IEEE 802 EC SG on TV White Space. He is currently participating in IEEE 802.11 and IEEE 802.19 activities related to TVWS

HIROSHI HARADA is director of the Ubiquitous Mobile Communication Group at NICT. After joining the Communications Research Laboratory, Ministry of Posts and Communications, in 1995, he researched SDR, cognitive radio, and broadband wireless access systems on the microwave and millimeter-wave bands. He has filled important roles for ITU-R WP5A, IEEE 802.15.3c/15.4g, IEEE 1900.4/6, and ETSI-RRS. He serves currently on the board of directors of the Wireless Innovation Forum (SDR forum), and as Vice Chair of IEEE 1900.4 and Chair of IEEE SCC41.

MÉRouAne DebBaH entered the Ecole Normale Superieure de Cachan, France, in 1996 where he received his M.Sc. and Ph.D. degrees in 1999 and 2002. He is presently a professor at Supelec, Gif-sur-Yvette, France, holder of the Alcatel-Lucent Chair on Flexible Radio. His research interests are in information theory, signal processing, and wireless communications.

KARI KALLIOJÄRVI [M] received his M.Sc. and Dr.Tech. degrees in electrical engineering from Tampere University of Technology in 1990 and 1995, respectively. Since 1995 he has been affiliated with Nokia Research Center, where he currently works as a principal researcher. His current research interests are related to wireless systems research and cognitive radio systems.

PAUL BENDER received his diploma degree in telecommunications in 1991. He joined the German Federal Office for Posts and Telecommunications (BAPT) in Mainz, Germany, where he was responsible for the standardization and elaboration of type approval specifications for cordless communication systems. In BNetzA, he is now responsible for the technical regulation of third generation mobile systems and systems beyond. In ICT-E $E^{3}$ and IST-E R I and II he worked on regulation issues of reconfigurability (e.g. responsibility chain) and flexible spectrum management.

Benoist Deschamps has a Ph.D. in electronics of telecommunications (specialization in microwaves passed with highest distinction) from ENST de Bretagne in 1998. He graduated as an engineer in telecommunications from ENST de Bretagne in 1993 (final year in UPC). He works at ANFR since 1998 as an expert in spectrum planning in the Spectrum Planning and International Affairs Division.

SITHAMPARANATHAN KANDEEPAN [SM] did his undergraduate studies in Colombo, and Master's in engineering (telecoms) and Ph.D. in engineering at the University of Technology, Sydney in 1999 and 2003 respectively. He is currently a senior researcher and leads the Cognitive Information Networks group at CREATE-NET, Italy. He is also an adjunct academic with the Australian National University, and held positions with the CRC-Satellite Systems, Sydney (2000-2004) and NICTA, Canberra (2004-2008).

MICHAEL STREET is principal scientist-communication systems at the Chief Technology Office, NATO C3 Agency. He began writing software radios in 1992 during a Ph.D. studying adaptive behavior for HF radio networks. He has since worked in academe, industry, and government, joining NC3A in 1999 and leading NATO work on SDR, security, and spectrum use at NC3A since 2004

Thomas Haustein received a Dr.-Ing. (Ph.D.) degree in mobile communications from the Berlin University of Technology, Germany, in 2006. In 1997 he was with the Fraunhofer Heinrich Hertz Institute (HHI), where he worked on wireless infrared systems and radio communications with multiple antennas and OFDM. He focused on real-time algorithms for baseband processing and advanced multiuser resource allocation. From 2006 till 2008 he was with Nokia Siemens Networks, where he conducted research for LTE and LTEAdvanced. He is currently head of the Broadband Mobile Communications Department at Fraunhofer HHI.

JASWINDER LOTA [SM] joined the University of East London as a senior lecturer in June 2009. Before that he was with Sepura plc. Cambridge United Kingdom, as a senior technologist, working on developing new technologies, algorithms, and standards relevant to future product development for TETRA mobile radios. Prior to joining Sepura, he was with the Navy from 1989 in various roles in engineering, research, and management, mainly pertaining to radar and EW systems. His research interests include delta-sigma modulators, wireless, and working towards ETSI TC RRS activities.

AAWATIF HAYAR received her M.Sc. degree from ENS, Cachan, France, in 1992 and her Specialization degree in communications systems and networks from ENSEEIHT-Toulouse France, in 1997. She received with honors her Ph.D. degree in Signal Processing and Communications from Institut National Polytechnique, Toulouse, in 2001. She is currently research and teaching associate with the Department of Mobile Communications at EURECOM. Her research interests include arrays of UWB systems, blind sensing algorithms, spectrum pooling strategies, and architecture design for cognitive radio systems. 